\title{
BMJ Global Health How primary healthcare workers obtain information during consultations to aid safe prescribing in low-income and lower middle-income countries: a systematic review
}

Chris Smith (D) , ${ }^{1,2}$ Michelle Helena van Velthoven, ${ }^{1,3}$ Nguyen Duc Truong, ${ }^{4,5}$ Nguyen Hai Nam (D) , ",6 Vũ Phan Anh, ${ }^{4,7}$ Tareq Mohammed Ali AL-Ahdal,, Osama Gamal Hassan, ${ }^{4,9}$ Basel Kouz, ${ }^{4,10}$ Nguyen Tien Huy, ${ }^{4}$ Malcolm Brewster, ${ }^{11}$ Neil Pakenham-Walsh ${ }^{11}$

To cite: Smith C,

van Velthoven MH, Truong ND, et al. How primary healthcare workers obtain information during consultations to aid safe prescribing in low-income and lower middle-income countries: a systematic review. BMJ Global Health 2020;5:e002094. doi:10.1136/ bmjgh-2019-002094

Handling editor Stephanie M Topp

- Additional material is published online only. To view please visit the journal online (http://dx.doi.org/10.1136/ bmjgh-2019-002094).

CS and MHvV are joint first authors.

Received 18 0ctober 2019 Revised 18 February 2020 Accepted 28 February 2020

Check for updates

(C) Author(s) (or their employer(s)) 2020. Re-use permitted under CC BY-NC. No commercial re-use. See rights and permissions. Published by BMJ.

For numbered affiliations see end of article.

Correspondence to

Dr Chris Smith;

christopher.smith@|shtm.ac.uk

\section{ABSTRACT}

Background We systematically reviewed the evidence on how primary healthcare workers obtain information during consultations to support decision-making for prescribing in low and lower middle-income countries.

Methods We searched electronic databases, consulted the Healthcare Information For All network, hand searched reference lists, ran citation searches of included studies and emailed authors of identified papers. Two reviewers extracted data and appraised quality with relevant tools. Results of 60497 records found, 23 studies met our inclusion criteria. Fourteen studies were observational and nine were interventional. Frequently mentioned sources of information were books, leaflets, guidelines, aids and the internet. These sources were sometimes out of date and health workers reported being confused which to use. Internet access varied and even when it was available, use was limited by technical issues. Of the five electronic tools that were assessed, four had positive outcomes. Tools assisted prescribers with medicine selection and dosage calculations, which increased prescribing accuracy. The quality of reporting varied but was overall low.

Discussion Studies indicated a lack of up-to-date and relevant medicine information in low and lower middleincome settings. Internet-based sources appeared to be useful when it is possible to download content for offline use and to update when there is internet access. Electronic tools showed promise, but their accuracy needs to be validated and they should focus on giving actionable advice to guide prescribers. PROSPERO registration number CRD42018091088.

\section{BACKGROUND}

Consultations by primary healthcare workers in low and middle-income countries make up the majority of healthcare interactions globally, yet little is known about how healthcare workers access information during

\section{Key questions}

What is already known?

- While there is a large amount of health information available, it is unclear to what extent primary healthcare workers access such information to aid prescribing in low and lower middle-income countries. Previous work found that digital health tools targeting primary healthcare workers had not been extensively studied.

What are the new findings?

- To our knowledge, this is the largest ever systematic review on how primary healthcare workers obtain health information during consultations to support decision-making for prescribing in low and lower middle-income countries. While included studies were of low quality, they indicated a lack of upto-date and relevant medicine information which makes it challenging for prescribers in low and lowe middle-income settings to access information.

What do the new findings imply?

- This study indicates a great need for high-quality studies to understand the prescribing information needs of primary healthcare workers. Internet-based sources appear to be useful when it is possible to download content for offline use and to update when there is internet access. Electronic tools show promise, but their accuracy needs to be validated and they should focus on giving actionable advice to guide prescribers.

consultations. This is important because it can be challenging for healthcare professionals to keep up to date with the expansion in medical knowledge and updated guidelines and recommended treatment regimens. These challenges could be further 
compounded in low and middle-income countries with less robust continuing professional development systems and more limited internet access. The WHO Medicines Report 2011 reports that 'Globally, most prescribers receive most of their prescribing information from the pharmaceutical industry and in many countries, this is the only information they receive. ${ }^{1}$ Unsafe and inappropriate prescribing is a huge problem, especially in low and middle-income countries, where many patients are given at least one drug per consultation. ${ }^{2}$ Antimicrobial resistance is a rising global health threat caused by the overuse of antibiotics. 'Rational' use of medicines depends largely on the ability of the health worker to make the correct diagnosis and then treat accordingly. ${ }^{4}$ Previous research has reported various issues related to inappropriate and unsafe prescribing. One of these issues is medication errors, meaning errors of dose or route of administration, and to errors of communication between prescriber and dispenser. ${ }^{56}$ Also, healthcare workers can prescribe the wrong medicine, with or without a failure to correctly diagnose the case. Also there is a wide variation in the availability and quality of health information and relevance to different settings with respect to their language, geographical focus and technical level. ${ }^{4}$ Outof-date offline resources might not have incorporated changes in medical knowledge and guidance that have occurred over time, such as new antibiotic regimen and diagnostic tests. When healthcare workers act on incorrect information this can lead to incorrect diagnosis and inappropriate prescribing. ${ }^{7}$

With 'healthcare information' we mean information that guides healthcare workers to prescribe at the point of care. This includes guiding them whether to prescribe a medicine, which medicine and details of the dose, route of administration, frequency of administration and duration of treatment. ${ }^{4}$ Examples of healthcare information resources are point-of-care decision tools, formularies, books, manuals, guidelines and protocols, rather than routine health information such as patient history, records and local epidemiological reports. This includes free and for-purchase materials, both in digital and offline forms.

To guide correct prescribing (including diagnosis and, where appropriate, selection of medicine), correct information is important as point-of-care information for consultations. Factors influencing whether healthcare providers attempt to access information during consultations include previous training and availability of the information, which could include internet access. ${ }^{4} 89$ Additional influencing factors might be how common or rare a condition is, the prescriber's familiarity with the treatment in question, whether it is a condition where the consequences of prescribing errors could be severe (eg, high risk of adverse effects, or if child dosing required), if the patient is being treated for comorbidities and a risk of drug interaction is suspected, and level of awareness of the need for information.
A previous systematic review found that digital health tools targeting primary healthcare workers had not been extensively studied. ${ }^{10}$ While there is a large amount of health information available, it is unclear to what extent primary healthcare workers access such information to aid prescribing. Therefore, this review aims to review the evidence on how primary healthcare workers obtain health information during consultations to support prescribing decision-making in low and lower middleincome economies.

\section{METHODS}

A protocol of this systematic review was previously published $^{11}$ and follows the Cochrane Collaboration ${ }^{12}$ and Centre for Review and Dissemination ${ }^{13}$ methodology for conducting systematic reviews where possible. The Preferred Reporting Items for Systematic Reviews and Meta-Analyses (PRISMA) was used for reporting (online supplementary file 1$).^{14}$

\section{Criteria for considering studies}

We included observational studies, such as cross-sectional surveys, cohort studies, qualitative studies (eg, interview studies and focus groups), mystery client studies, and intervention studies, such as randomised controlled trials (RCT) and non-randomised studies (eg, controlled studies, before-and-after studies, interrupted time series studies). We only included studies reported in English that were published after 2000 to provide up-to-date and relevant evidence. ${ }^{11}$

We included studies involving primary healthcare workers in low and lower middle-income countries who prescribe or dispense medication or order medical tests, such as doctors, clinical officers, nurses, midwives, pharmacists, drugstore vendors (with varying qualifications) or community health workers. Primary healthcare workers encompass a diverse range of healthcare cadres such as doctors, clinical officers, nurses, pharmacists and drugstore vendors and are often the first level of contact of individuals, the family and the community with the national health system. ${ }^{15}$ We excluded studies focusing on secondary care or hospital settings, allied health professionals, for example, physiotherapists and traditional medicine health workers. ${ }^{11}$

We included any interventions to improve access to healthcare information during a consultation for prescribing, for example, tools and aids (possible interventions could use digital resources, books, protocols, and so on). Also, studies that did not focus on an intervention (eg, observational studies) were included. We did not include studies focusing on interventions that aimed to improve the quality of care, supervision or mentoring of healthcare workers unless they also included tools or aids for healthcare workers that were available during the consultation. We included any type of comparator interventions and studies that did not use a comparator. ${ }^{11}$ 
The primary outcome of this review is the proportion of healthcare workers obtaining healthcare information during consultations or transactions (ie, from the time when the patient arrives to leaving) from different sources (eg, use of books, guidelines, digital resources, peer networks, no information-memory). Secondary outcomes are any change in healthcare provider knowledge or behaviour (eg, prescribing in intervention studies), clinical outcomes, adverse outcomes (eg, misconceptions resulting from out-of-date or incorrect information, whether obtaining information to guide prescribing relevant to a consultation affects the quality of a consultation; eg, patients trust a healthcare worker less or perceive a lack of empathy when a health worker looks up information) and use of resources. ${ }^{11}$

\section{Information sources and search strategy}

Relevant articles were identified by searching electronic databases: MEDLINE through Ovid; EMBASE through Ovid; CABI Global Health through Ovid; WHO Global Health Library; POPLINE; Africa-Wide Information; Library, Information Science \& Technology D/base; Web of Science; Cochrane Central Register of Controlled Trials (CENTRAL); WHO International Clinical Trials Registry Platform; and ClinicalTrials.gov. In addition, the Healthcare Information For All (HIFA) network was consulted for relevant publications and emailed authors of identified papers to identify additional articles. After identifying eligible studies, we ran citation searches of included studies (eg, in Google Scholar, Scopus or Web of Knowledge). ${ }^{11}$

Final search strategies were tailored to different databases with a medical research librarian (John Eyers). No study design filter was used as both quantitative and qualitative studies were included. We used the titles, abstracts and keywords of a set of articles for which we knew that met our inclusion criteria to define a search strategy that returned all these articles. ${ }^{11}$

\section{Data management, collection and analysis}

We excluded duplicate references by comparing titles, authors and digital object identifiers between similar search results using two software programs (EndNote and EPPI-Reviewer). Two reviewers screened titles and abstracts of search results against the inclusion and exclusion criteria. One reviewer retrieved a full-text paper when a study included participants who are primary healthcare workers in low or lower middle-income economies, and it assessed one or more relevant outcome measures. Two reviewers assessed full texts for eligibility, and any disagreement was resolved through discussion with a third author. ${ }^{11}$

Data were extracted from included studies using a standardised Excel form that included general information (title, authors, date, and so on), study characteristics (study design, aim, duration, inclusion and exclusion criteria), risk of bias (depending on study design), participants (description, geographic location setting, and so on), intervention (if appropriate and to include sources of online or offline information used), outcomes (as specified above, other outcomes, adverse events) and results (outcomes, times of assessment). We piloted the data extraction form on a small number of studies to develop the final data extraction form. Two reviewers extracted data from the included studies. ${ }^{11}$

Quality assessment was undertaken by two reviewers. Any disagreements were resolved by consensus and the opinion of a third reviewer. The methods specified in the Cochrane Collaboration tool for assessing the risk of bias were used. Three bias assessment categories were used: low, high and unclear risk, as specified in the Cochrane Collaboration Handbook. ${ }^{12}$ For other types of studies we used adapted versions of the following: the Cochrane Recommend tool for assessing Risk of Bias in Non-randomised Studies of Interventions ${ }^{16}$; the Critical Appraisal Skills Programme tool for qualitative studies ${ }^{17}$; and the Appraisal tool for Cross-Sectional Studies (AXIS). ${ }^{18}$

We provide a narrative overview of findings and tabular summaries of extracted data. It was not possible to perform a meta-analysis due to differences in study populations, interventions and outcomes. ${ }^{11}$

We provide a narrative overview of subgroups including different study types, interventions (eg, digital vs analogue), cadres of healthcare workers and geographic regions. We use the term 'observational studies' for any type of study where the investigators asked about the use of information sources but did not deliver training or introduce a particular source of information or tool. We use the term 'intervention studies' for any type of study that assessed an intervention, such as RCTs, before-andafter studies, comparison studies and evaluations of information tools. ${ }^{11}$

\section{Patient and public involvement}

The broader HIFA network was consulted for comments and suggestions at various stages during the systematic review. Patients were not involved in the design or analysis of this review. ${ }^{11}$

\section{RESULTS \\ Results of the search}

We conducted searches in February 2018 and found 60497 records after removing duplicates (figure 1). Teams of two reviewers screened the 60497 records and narrowed this down to 993 records which were then screened again by two reviewers (MHvV and NTH). We assessed 127 fulltext articles for eligibility; whether they involved primary healthcare workers in low or lower middle-income economies who prescribe and/or dispense medication. We included 19 studies on which we conducted citation searches in July 2019 which resulted in finding a further four studies. Finally, 23 studies met our inclusion criteria: 14 observational studies shown in table 1 , and 9 intervention studies shown in table 2 . 


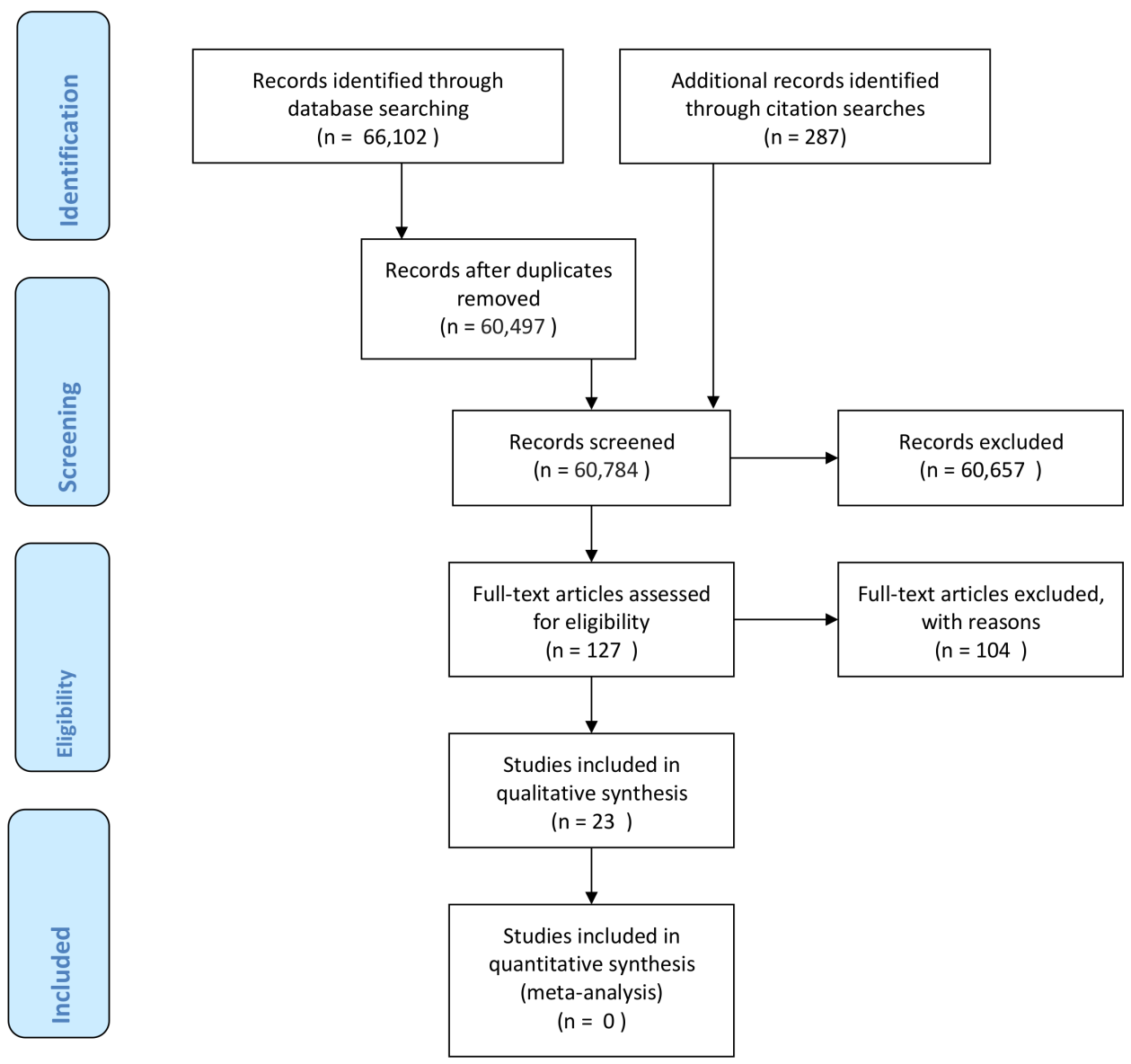

Figure 1 Preferred Reporting Items for Systematic Reviews and Meta-Analyses (PRISMA) 2009 study flow diagram.

\section{Description of studies}

Table 1 shows that of the 14 included observational studies, nine used surveys of which three additionally used qualitative methods. The other five observational studies were qualitative studies of which three used interviews and two focus groups. Study samples varied from 12 to 192. Eleven studies were conducted in Africa, two in Asia and one in Oceania. Studies took place in pharmacies, or health facilities and involved pharmacists, health workers and community health workers.

Table 2 shows that of nine included intervention studies, there was one cluster RCT, one study comparing tools without randomisation, two before-and-after studies and four studies evaluating the development and/or implementation of a tool. Two intervention studies were part of the larger 'Algorithm for Management of Childhood Illness' (ALMANACH) project and two studies assessed the medication dosing app using 'CommCare'. Four studies used quantitative, two qualitative and three mixed methods. Study samples varied from 3 to 3914. Six studies were conducted in Africa, one in Asia and two in South America. Studies took place in health facilities and involved health workers and/or community health workers.

We excluded 104 full-text studies with reasons provided (online supplementary file 2 ), mostly because they were not about information seeking during the consultation.

\section{Quality assessment of included studies}

Among the observational studies the best reported domain was a clear statement of the aim of qualitative and cross-sectional studies. For qualitative studies, the relationship between the researcher and the participants was often unclear. For both qualitative and cross-sectional studies, the rationale for choosing the specific method was frequently not clearly stated. Also, in survey studies, the issue of non-responders was often not appropriately addressed (online supplementary file 3 ).

Among the intervention studies, the cluster RCT reported using block randomisation but did not mention allocation concealment. Other intervention studies were likely to suffer from performance and detection bias and other biases due to small sample sizes (online supplementary file 3 ).

\section{Primary outcome in observational studies}

All observational studies reported on the use of healthcare information during consultations, which is reported in table 3 for nine studies involving a survey and in table 4 for the five qualitative studies. The most mentioned information sources used during consultations were books, ${ }^{719-23}$ leaflets, ${ }^{20}$ guidelines, ${ }^{720} 21$ 24-28 a flip chart and reporting form aid, ${ }^{29}$ and the internet. ${ }^{72124263031} \mathrm{~A}$ survey conducted in Harare, Zimbabwe, in 2006 found that 28 out of 46 community pharmacists $(61 \%)$ used 


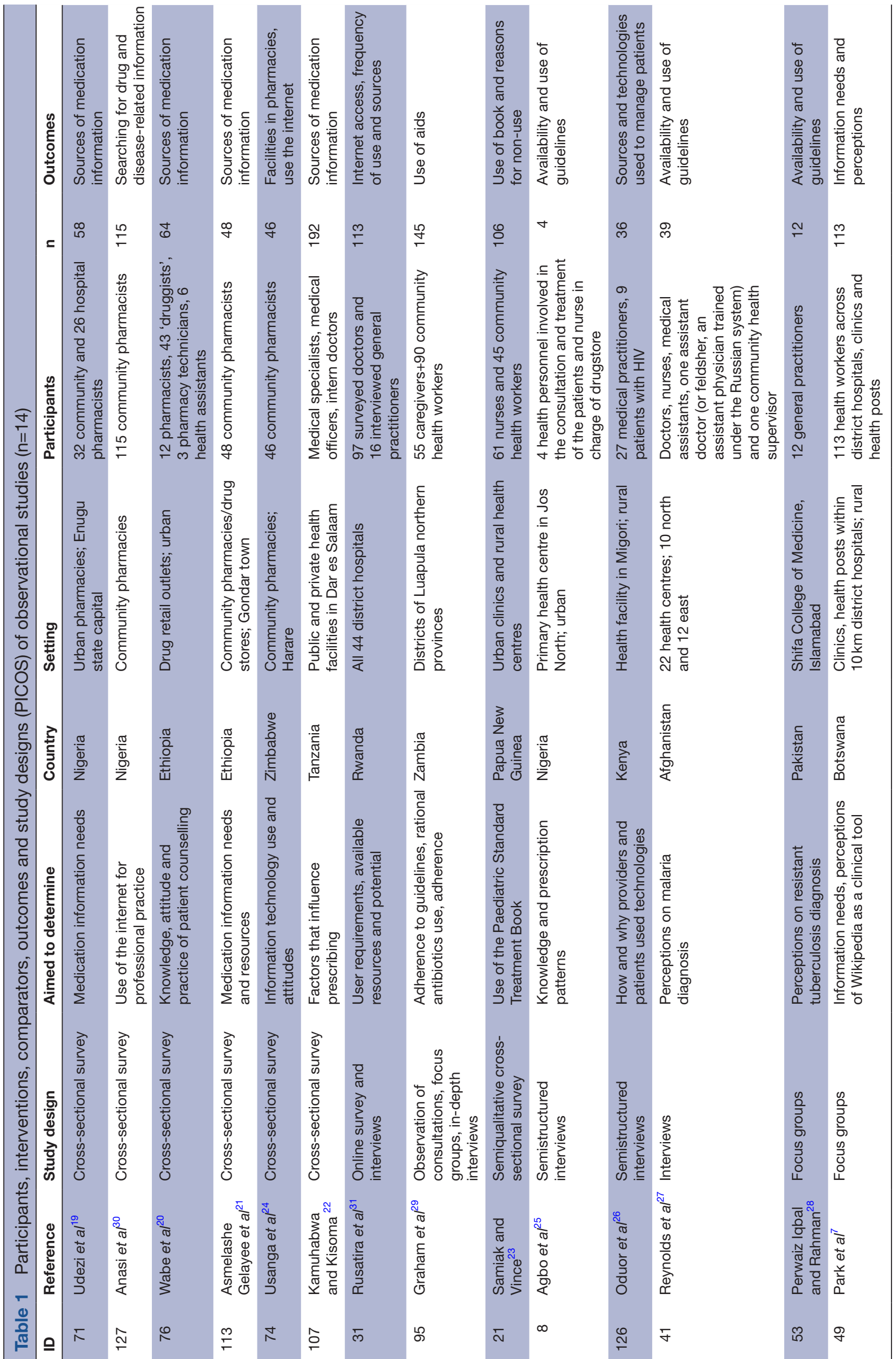

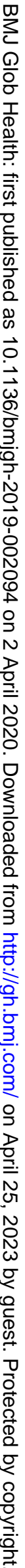




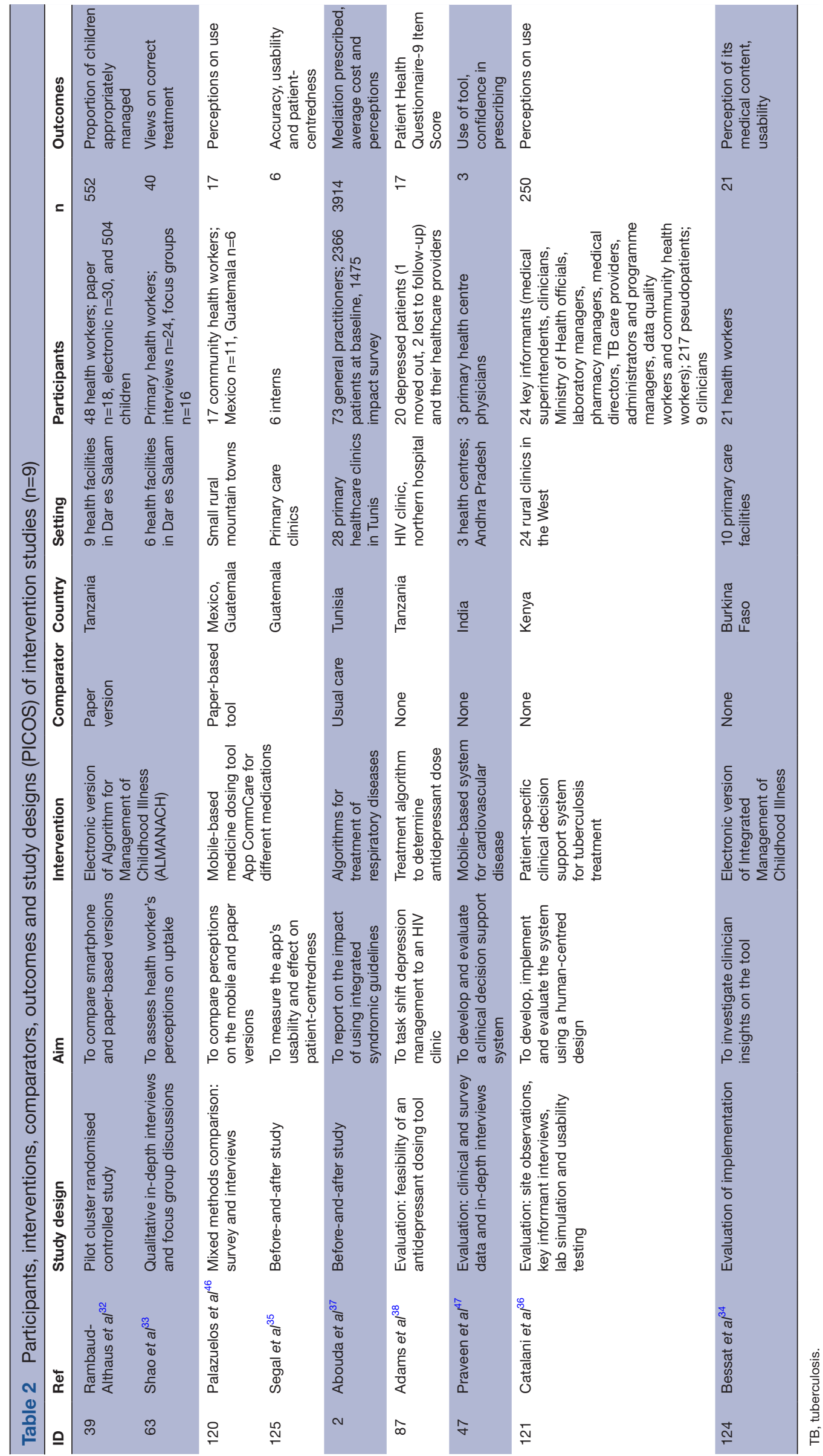




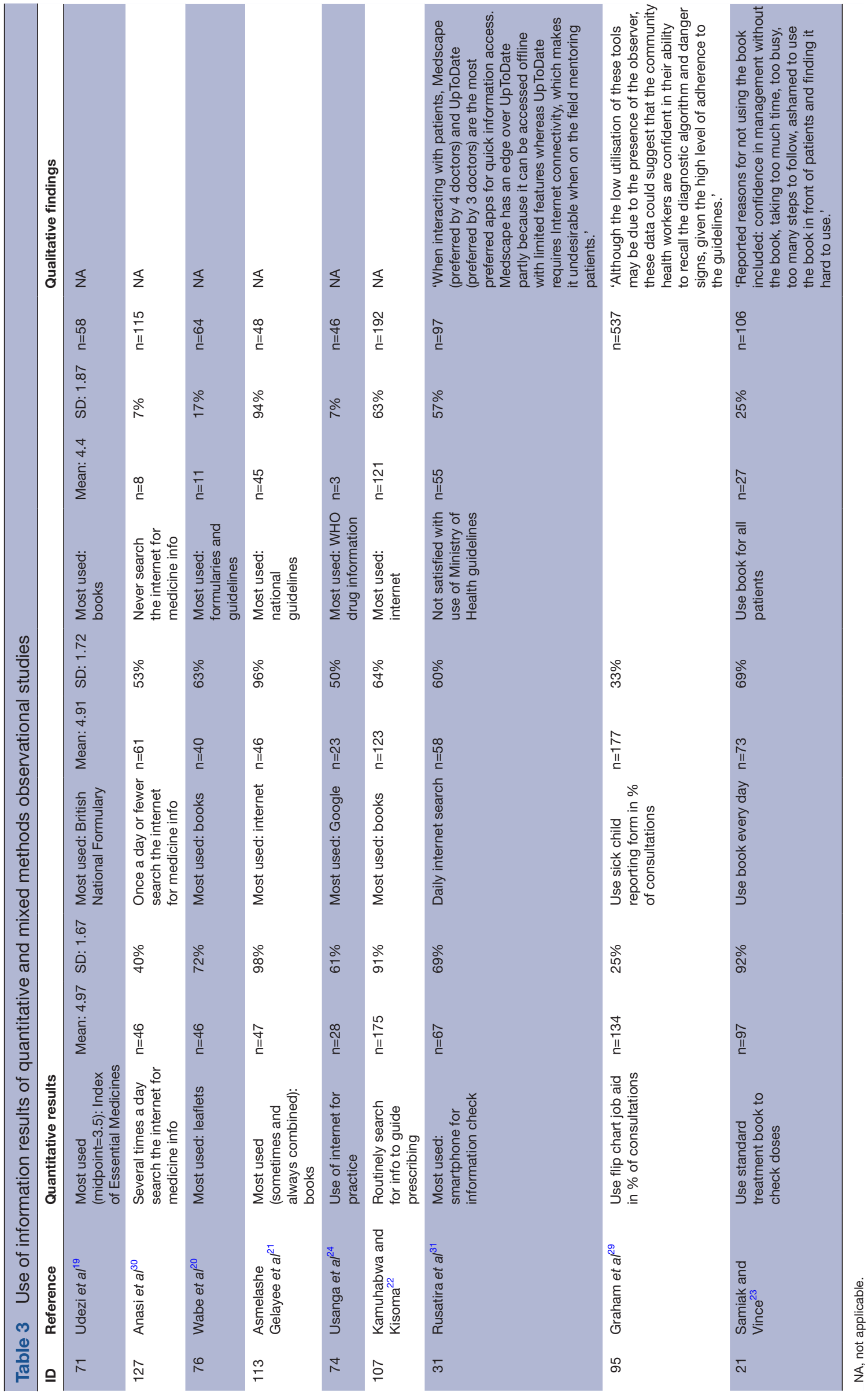

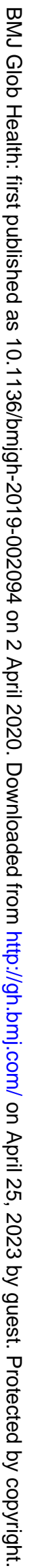


Table 4 Use of information results of qualitative observational studies

\begin{tabular}{cll}
\hline ID & Reference & Qualitative findings \\
\hline 8 Agbo et al ${ }^{25}$ & 'No copy of the National policy guideline on malaria treatment had been seen or used by the health personnel and \\
none had ever attended any training in form of workshop or seminar on malaria.' \\
'Health workers identified challenges to efficient work as inadequate manpower, lack of materials like current national \\
policy guidelines on disease management especially those commonly seen at the grassroots, lack of blood pressure \\
measuring apparatus, erratic power supply, dilapidated structures and inadequate training opportunities.' \\
'Clinicians reported the use of various sources of information as they looked up drug information, guidelines, and other \\
medical content required to support patients. For example, a clinician described the tools he used to learn more about \\
new drugs when asked about his source of information regarding recommended drugs: "My information sources are \\
many. For example, I can read about new drugs from government circulars or from MEDS (Mission for Essential Drugs \\
and Supplies) who are also ISO certified and have a laboratory where they conduct rigorous tests on their drugs. \\
MEDS is accessible online [via desktop computers or mobile phones]".' (P2, Clinician) \\
'Clinicians also relied on various information sources to make decisions on which drugs to prescribe. These included \\
the official government drug index booklet (Drug-Index.IT), the Medscape website, the OMNIO medical resources \\
website and Ministry of Health guidelines.'
\end{tabular}

\begin{tabular}{|c|c|c|}
\hline 41 & Reynolds et $a l^{27}$ & $\begin{array}{l}\text { 'Some participants referred to guidelines when describing what they would prescribe to a patient with malaria, } \\
\text { particularly those patients considered to be special; for example, young children or pregnant women. Thus, it appeareo } \\
\text { that guidelines were largely interpreted as tools to inform the prescription of medicines following a malaria diagnosis, } \\
\text { rather than to inform the process of diagnosis itself.' }\end{array}$ \\
\hline 53 & $\begin{array}{l}\text { Perwaiz lqbal and } \\
\text { Rahman }^{28}\end{array}$ & $\begin{array}{l}\text { 'Most of the general practitioners were not aware of screening guidelines for close contacts of Tuberculosis patients. } \\
\text { The WHO guidelines for Management of Tuberculosis are used in South Asian countries. However, the Pakistan Chest } \\
\text { Society has published its own guidelines under the name of National Tuberculosis Guidelines, which are different from } \\
\text { WHO. The general practitioners were using one or the other and somewhat confused as to which guidelines to follow.' }\end{array}$ \\
\hline
\end{tabular}

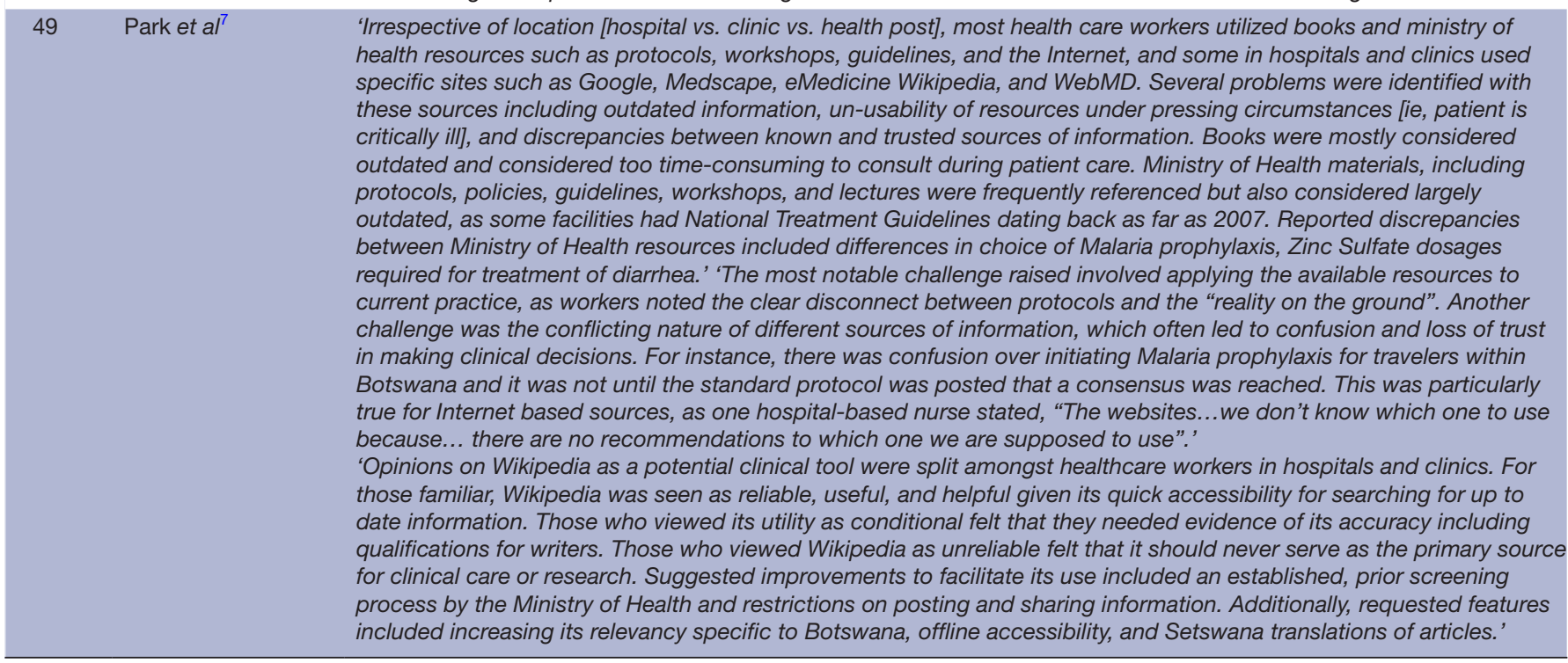

the internet for practice and $23(50 \%)$ mentioned using Google. ${ }^{24}$ A more recent survey in Nigeria in 2016 found that 46 out of 115 community pharmacists (40\%) searched the internet several times a day for drug information and only $8(7 \%)$ never searched the internet. ${ }^{30} \mathrm{~A}$ study observing community health worker consultations with children who had suspected pneumonia in Zambia found low use of the flip chart and reporting form aid and suggested this might be because community health workers were confident in their recall of the diagnostic integrated community case management algorithm. ${ }^{29}$ Reasons for not using books were that it took too much time to use them, ${ }^{723}$ prescribers felt confident in their decisions, were too busy or felt ashamed to use it in front of patients. ${ }^{23}$ Health workers participating in interviews in Afghanistan used malaria guidelines for prescribing medication ${ }^{27}$ while in Nigeria, these were not available and this was a challenge for health workers to work efficiently. ${ }^{25}$ Health workers participating in focus groups in Botswana (published 2016) considered the available guidelines and other Ministry of Health materials were outdated and were confused about which internet-based sources to use. ${ }^{7}$ General practitioners participating in focus groups in Pakistan were also confused regarding the use of international or national tuberculosis guidelines. $^{28}$

In terms of secondary outcomes in observational studies, four studies reported on resource-related outcomes, one on behaviour and one on knowledge (online supplementary file 4). A survey in Zimbabwe found that 28 out of $46(61 \%)$ pharmacies had internet access, 33 had networked computers $(72 \%)$ and 3 access to electronic databases (7\%). ${ }^{24} \mathrm{~A}$ survey in Ethiopia (undertaken in 2009) found that only 7 out of 48 (15\%) pharmacies had internet access. ${ }^{21}$ Forty-nine out of $97(51 \%)$ general practitioners in hospitals in Rwanda had access to Wi-Fi 
in their hospitals (year of research unstated; published 2016) ${ }^{31}$ Focus groups in Botswana found that while many facilities had internet access, use was limited because of 'technical installation issues, connection, password access, costs, lack of time, and lack of devices'. ${ }^{7}$ Ninetythree out of 106 health workers $(88 \%)$ in Papua New Guinea had the Paediatric Standard Treatment Book with them. ${ }^{23}$ Twenty-eight out of 64 pharmacy workers (44\%) in Ethiopia said they lacked the knowledge for rational prescribing and needed updated information. ${ }^{20}$

\section{Secondary outcomes in interventional studies}

All nine intervention studies reported on health worker behaviour-related outcomes (table 5). The cluster RCT in Tanzania reported significantly higher $(\mathrm{p}<0.001)$ proportions of children appropriately managed in the two intervention arms: $62 \%$ in paper (range: $55 \%-74 \%$, $\mathrm{n}=171$ ); and $63 \%$ in the electronic arm (range: $52 \%-72 \%$, $\mathrm{n}=167$ ), compared with $37 \%$ in the control arm (range: $29 \%-44 \%, n=166) .^{32}$ The accompanied qualitative study revealed that the algorithm (ALMANACH) helped health

Table 5 Behaviour-related results of interventional studies

\begin{tabular}{|c|c|c|}
\hline ID & Author & Behaviour-related findings \\
\hline 39 & $\begin{array}{l}\text { Rambaud-Althaus } \\
\text { et al }\end{array}$ & $\begin{array}{l}\text { 'The proportion of children appropriately managed (antimalarials, antibiotics, zinc, and rehydration prescribed when } \\
\text { needed only) was similar in the two intervention arms: } 62 \% \text { in paper (range: } 55 \%-74 \%, n=171) ; \text { and } 63 \% \text { in the } \\
\text { electronic arm (range: } 52 \%-72 \%, n=167) \text {. The proportion of children appropriately managed was significantly lower } \\
(p<0.001) \text { in the control arm }(37 \%, \text { range: } 29 \%-44 \%, n=166) \text { than in the paper }(R R=1.7[1.3-2.2]) \text { and electronic arm }(1 . \\
[1.3-2.2]) \text {.' }\end{array}$ \\
\hline 63 & Shao et $a l^{33}$ & $\begin{array}{l}\text { 'Health workers pointed out that the ALMANACH assisted them to reduce antibiotic and antimalarial prescription as the } \\
\text { device walked them step-by-step through the consultation starting from diagnosis to treatment including calculation } \\
\text { of proper dosage of required drugs. Thus, the majority of the study participants (10 smartphone/11 tablet) stated that } \\
\text { both devices reduced antibiotic prescription compared to routine practice. "Yes, before I was prescribing antibiotics } \\
\text { as antibiotics, I was just prescribing antibiotics, but truly now you don't believe, now I know many diseases are febrile } \\
\text { diseases, they don't need antibiotics".' (IDI, female, smartphone, very high uptake) } \\
\text { 'More than half of the respondents (8 smartphone/7 tablet) highlighted that the ALMANACH enabled correct treatment } \\
\text { "There are many advantages; first, the phone is a reference point in the sense that if you have forgotten what the patier } \\
\text { is suffering from, or treatment or medication, by following the instructions in the phone you will know the diagnosis ano } \\
\text { medicine to that diagnosis. So the phone helps a lot".' (IDI, male, smartphone, very low uptake) }\end{array}$ \\
\hline
\end{tabular}

\begin{tabular}{|c|c|c|}
\hline 120 & Palazuelos et $a l^{46}$ & $\begin{array}{l}\text { 'Use of the mHealth tool generally resulted in more accurate answers when compared to the paper-based tool. For } 6 \text { of } \\
7 \text { practice test questions, the mean score among those who answered with the mHealth tool was notably higher than } \\
\text { the mean score among respondents who answered with the paper-based tool. In general, the difference was greatest } \\
\text { in the questions that asked for pediatric doses based on age and weight, as opposed to standardized doses and } \\
\text { courses for adults. Although not coded nor quantified, the majority of the errors with each tool followed a few general } \\
\text { themes. For the paper-based tool, the community health workers often found it challenging to find the } 3 \text { different dosing } \\
\text { elements needed (dose, schedule, and duration) as they were often in disparate locations without any clear pattern } \\
\text { to follow. For the mHealth tool, the community health workers produced a wrong result if they inadvertently entered } \\
\text { information incorrectly at some stage of the algorithm (ie, if they entered in a wrong gender, age, weight, etc).' } \\
\text { 'Overall, the CHWs in both countries accepted the mHealth tool as a satisfactory tool that was appropriate for use in } \\
\text { dosing a medicine. Some CHWs noted that using the mHealth tool on a phone would be a way to gain credibility in the } \\
\text { community. The people, upon seeing us look in the book, think badly of us. With the phone, they think we are important. } \\
\text { The phone is a more acceptable way to access information in front of the patient so as to not lose face.' }\end{array}$ \\
\hline 2 & Abouda et $a l^{37}$ & $\begin{array}{l}\text { 'The number of drugs prescribed per patient who received drug prescription decreased by } 18.8 \% \text { in the impact survey } \\
\text { (3.2 vs 2.6,p<0.001).' }\end{array}$ \\
\hline 125 & Segal et $\left.a\right|^{35}$ & $\begin{array}{l}\text { 'Dosing accuracy improved from } 64.7 \% \text { (among } 156 \text { prescriptions) to } 92.4 \% \text { (among } 210 \text { prescriptions) when providers } \\
\text { used the app. Dosages prescribed after implementation were } 40 \% \text { more likely to be correct (relative risk: } 1.39 ; 95 \% \mathrm{Cl} \\
1.16 \text { to } 1.68 ; p=0.0005 \text { ). All providers appeared to dose medications more accurately after the intervention.' }\end{array}$ \\
\hline 87 & Adams et $a l^{38}$ & $\begin{array}{l}\text { 'The study nurse correctly identified all algorithm-indicated antidepressant recommendations }(n=74,100 \%) \text { and } \\
\text { communicated all to the study clinical officer.' }\end{array}$ \\
\hline 47 & Praveen et $a l^{47}$ & $\begin{array}{l}\text { 'Among those not on medications, } 31 \%(11 / 36) \text { were recommended for treatment by the decision support tool. The } \\
\text { physician commenced all these patients }(n=11) \text { on BP-lowering treatment.' }\end{array}$ \\
\hline 121 & Catalani et $a l^{36}$ & $\begin{array}{l}\text { 'Although providers rated the messages relatively highly, they found the accuracy and actionability of the clinical } \\
\text { decision support system problematic. Providers indicated that roughly over a quarter of the reminders were not correct } \\
\text { for that particular patient and that particular day. Moreover, slightly less than half of the reminders were not considered } \\
\text { actionable on that day.' }\end{array}$ \\
\hline 124 & Bessat et $a l^{34}$ & $\begin{array}{l}\text { 'Positive effects were mentioned to be better management of children ( } 5 \text { IDI, } 1 F G D) \text {, facilitation in treatment decision- } \\
\text { making and dosage calculation (7IDI, } 1 F G D) \text {, standardization of treatment (2IDI) and rational use of medicines (6IDI). The } \\
\text { application guides the clinician trough the assessment of the child up to the treatment and the counselling part. At the } \\
\text { end, a free text question gives room to the clinician to add additional classifications and treatments. Half of the study } \\
\text { participants reported not to add an antibiotic when the application did not recommend it, and mentioned it helped them } \\
\text { to rationalize the use of drugs. However, the other half of the participants (6IDI, } 1 F G D) \text { admitted to sometimes add an } \\
\text { antibiotic even though the application did not recommend it. Reasons mentioned were: to calm or treat cough (5IDI), to } \\
\text { prevent re-consultation (2IDI, } 1 F G D), \text { to cover severe diseases or prevent worsening of the disease (3IDI) and in cases of } \\
\text { fever with a negative malaria RDT result (1IDI, 1FGD).' }\end{array}$ \\
\hline
\end{tabular}

ALMANACH, Algorithm for Management of Childhood Illness; BP, blood pressure; CHW, community health worker; FGD, focus group discussion; IDI, in-depth interview; RDT, rapid diagnostic test; RR, relative risk. 
workers to reduce prescriptions as the device provided them with calculations and appropriate dosing of the required medication. ${ }^{33}$ A qualitative study in Burkina Faso also found that an electronic Integrated Management of Childhood Illness tool helped half of the health workers to rationalise the use of drugs. However, the other half prescribed an antibiotic-even when the application did not recommend it-in the belief that this might calm or treat cough, prevent patients from returning or prevent worsening of the disease. ${ }^{34}$ A before-and-after study in Guatemala found dosing accuracy to improve from $65 \%$ (among 156 prescriptions) to 92\% (among 210 prescriptions) when interns used a medication dosing app. ${ }^{35}$ The accuracy and actionability of a tuberculosis clinical decision support system were found problematic in an evaluation in Kenya. ${ }^{36}$

Three intervention studies reported on knowledge of health workers (online supplementary file 4). A beforeand-after study in Tunisia found that all general practitioners said the integrated syndromic disease guidelines had improved their knowledge after training but thought the translation of symptoms in Arabic to French could be confusing. ${ }^{37}$ An evaluation in Kenya found that clinicians had insufficient knowledge about isoniazid preventive therapy for tuberculosis and therefore simple alerts or reminders were inadequate without information on which steps to take. ${ }^{36}$ The qualitative study accompanying the RCT found that rational judgement was not compromised when using the ALMANACH. ${ }^{33}$

Two intervention studies reported on a patient outcome (online supplementary file 4). A feasibility study in Tanzania on an antidepressant medication dosing tool found the average Patient Health Questionnaire-9 Item Scores among 17 completers significantly decreased from 20 (SD: 3) at baseline to 8 (SD: 2$)$ at week $12(\mathrm{p}<0.001) .^{38}$ A before-and-after study of a medication dosing app in Guatemala observed no change in patient-centredness. ${ }^{35}$ Another four studies reported on the use of resources (online supplementary file 4). Only one study mentioned costs explicitly; the average cost of drug prescription per patient who was prescribed any drug was reduced by $19.3 \%$ in the impact survey from 8.2 to 6.75 Tunisian dinars $(\mathrm{p}<0.001) . .^{35}$

\section{DISCUSSION}

\section{Summary of main results}

To our knowledge, this is the largest ever systematic review on how primary healthcare workers obtain health information during consultations to support decision-making for prescribing in low and lower middle-income countries. Of the 60497 records found, 23 studies met our inclusion criteria. Most studies were conducted in Africa $(n=17)$. Of 14 observational studies, nine used surveys of which three additionally used qualitative methods. The other five observational studies were qualitative studies of which three used interviews and two focus groups. Frequently mentioned sources of information were books, leaflets, guidelines, aids and the internet. Reasons for not using books included the excessive time to use them, prescribers felt confident in their decisions, were too busy or felt ashamed to use them in front of patients. Also, these sources were sometimes out of date and health workers were confused which ones to use, particularly for websites. Internet access varied across settings and even when available, use was sometimes limited because of technical issues. The nine intervention studies included one RCT and an accompanying qualitative study, one study comparing electronic and paper-based tools, two before-and-after studies and four studies evaluating the development and/or implementation of a tool. Of the five electronic tools that were assessed after training health workers how to use them in these studies, four were assessed positively. Medication tools helped health workers to appropriately prescribe by providing them with calculations for appropriate medicine doses, which increased prescribing accuracy. One tool was not found actionable. Also, medication was sometimes still prescribed when a tool did not recommend it in the belief that it might prevent patients from returning or prevent worsening of the disease.

\section{Strengths and weaknesses}

Our review followed, where possible, the Cochrane Collaboration and Centre for Review and Dissemination methodology for conducting systematic reviews and we reported our findings based on guidelines from PRISMA statement. This meant that a comprehensive search and assessment of the evidence was conducted. The interventions researched in the studies were diverse and took place in different countries. Therefore, it was not possible to conduct an appropriate meta-analysis.

Most studies took place in low and lower middle-income countries in Africa. Research has shown that there can be a difference between the medicines prescribed and those dispensed. ${ }^{39}$ Our review did not report on whether the medicine was dispensed. Few studies specifically aimed to assess how primary healthcare workers accessed information during consultations and more studies on this topic are required. Another limitation is that the term 'primary healthcare worker' is generalised and lacks distinction among levels of education and healthcare delivery, which in many studies was not clearly described. Community health workers often can only prescribe one malarial, antibiotic, oral rehydration solution and zinc, which they can prescribe using a syndromic approach. Primary healthcare workers at health centres may have a few more choices of medicines, but often also lack diagnostic tools to prescribe the correct medicine and dose, or not to prescribe at all. Particularly in the publicly funded lower levels of healthcare systems, healthcare workers usually can only prescribe a small number of medicines. They are usually able to prescribe medicines that can be purchased by patients at pharmacies, depending on the patient's ability to pay and availability of medicines which can be limited by stock-outs. In the private sector, practitioners 
and pharmacists and local drug distributors can prescribe in certain settings and are often keen on prescribing medicines that provide them the highest profit. ${ }^{40}$ While the effectiveness of the private sector is typically limited in low-income countries, patients still prefer to go there for different reasons, including that medicines in the private sector are more competitively priced and accessible than in the public sector. ${ }^{41}$ To improve prescribing in these settings, incentives and training are important to consider in addition to the availability of up-to-date and high-quality information. ${ }^{42}$

Furthermore, assessment of costs was very limited in the included studies. Only one study mentioned costs explicitly; the average cost of drug prescription per patient who was prescribed any drug was reduced by $19.3 \%$ in the impact survey from 8.2 to 6.75 Tunisian dinars $(p<0.001) .^{35}$ Another study conducted in Botswana ${ }^{7}$ found that while many facilities had internet access, use was limited because of 'technical installation issues, connection, password access, costs, lack of time, and lack of devices'.

\section{Quality of the evidence}

The quality of reporting varied and was low overall. We found only one RCT which reported using block randomisation but did not mention allocation concealment. Other intervention studies were likely to suffer from performance and detection bias, and other bias due to small sample sizes. The observational studies were reported with varying quality. It is crucial that future studies improve their methodology to enhance the strength of the evidence.

\section{Agreements and disagreements with other studies or reviews}

Unsafe prescribing by primary healthcare workers has been attributed to weak medication systems, poor environmental conditions or staff shortages, poor education, inadequate training and lack of knowledge and skills. ${ }^{43}$ Previous studies in Africa have suggested variations in knowledge about the basics of how to diagnose and manage common diseases are commonplace and often associated with suboptimal, ineffective and dangerous healthcare practices. ${ }^{48}$ One study in our review found that a considerable proportion of pharmacy workers $(44 \%)$ in Ethiopia said they lacked prescribing-related knowledge and needed updated information. ${ }^{20}$ An evaluation in Kenya found that clinicians had insufficient knowledge about isoniazid preventive therapy for tuberculosis and therefore simple alerts or reminders were inadequate without information on which steps to take. ${ }^{36}$

A cohort study on prescribing patterns of evidencebased heart failure medicine concluded that improved uptake of guidance for prescribing is necessary to improve patient outcomes. ${ }^{44}$ Another systematic review found that most of the interventions to improve healthcare worker performance have focused on 'supervision' as opposed to 'tools and aids' such as protocols and/or charts. ${ }^{10}$ Our review fills this gap by providing evidence on tools and aids. The most mentioned information sources used during consultations were books, ${ }^{79-23}$ leaflets, ${ }^{20}$ guidelines, ${ }^{7202124-28}$ a flip chart and reporting form aid, ${ }^{29}$ and the internet. 72124263031 The nine intervention studies assessed tools such as electronic versions of algorithms for the management of childhood illness and mobilebased medicine and dosing tools for different diseases (general, respiratory disease, depression, cardiovascular disease).

Prescribing rationally, therapeutically and safely becomes ever more complicated as the number of widely available medicines increases and medical knowledge expands. The WHO Model Essential Medicines List (EML) listed 212 medicines in its first edition in 1977 more than doubling to 460 medicines in the 2019 21st edition. ${ }^{45}$ The WHO considers the EML a 'flagship tool to expand access to medicines' which is necessary for the achievement of Sustainable Development Goal 3. Furthermore, in practice, the ELM only covers a small fraction of the medicines that are commonly available and used or misused worldwide. Up-to-date medicine information and the means of making it accessible and acceptable to prescribers at the point of care must accompany the expanding access to medicines if those seeking medical care are to benefit. Such information needs to go beyond information about individual medicines, to include guidance on the selection of medicines.

\section{CONCLUSION}

This systematic review found a small number of lowquality studies showing a lack of up-to-date and relevant medicine information resources in low and lower middleincome settings. Internet-based sources are useful when it is possible to download content for offline use and to regularly update when there is internet access. Given the fundamental importance of safe and effective prescribing, and its dependence on reliable information (whether retained through training or available at point of care), it is remarkable that the available research tells us little about the prescribing information needs of primary health workers, and how these needs can be progressively met. Electronic tools to help healthcare workers to prescribe medication showed promise, but their accuracy needs to be validated. Such tools should focus on actionable advice that guides prescribers through the different steps that need to be taken to prescribe safely.

Future work needs to improve quality of research methodology, provide a clear description of the context, assess costs and cost-effectiveness and consider interactions between the availability and use of information and other factors that influence prescribing. Future conduct of observational studies could provide evidence of what really happens at different levels of the health delivery system, which will likely vary substantially in different countries and even between regions of the same countries, as well as in response to different diseases. Changing drug protocols may need training and widespread 
information dissemination as well as an enabling environment to make change possible.

\section{Author affiliations}

${ }^{1}$ School of Tropical Medicine and Global Health (TMGH), Nagasaki University, Nagasaki, Japan

${ }^{2}$ Department of Clinical Research, London School of Hygiene and Tropical Medicine Faculty of Infectious and Tropical Diseases, London, UK

${ }^{3}$ Department of Paediatrics, Oxford University, Oxford, UK

${ }^{4}$ Nagasaki University, Nagasaki, Japan

${ }^{5}$ Obstetrics and Gynecologic Department, FV Hospital, Ho Chi Minh City, Vietnam

${ }^{6}$ Department of General Surgery, Ho Chi Minh City University of Medicine and Pharmacy, Ho Chi Minh City, Vietnam

${ }^{7}$ Department of Neonatology, Children's Hospital 2, Ho Chi Minh City, Vietnam

${ }^{8}$ Faculty of Medicine, Jordan University of Science and Technology, Irbid, Jordan

${ }^{9}$ Faculty of Medicine, South Valley University, Qena, Egypt

${ }^{10}$ Faculty of Medicine, Damascus University, Damascus, Damascus Governorate, Syria

${ }^{11}$ Healthcare Information For All (HIFA) network, Global Healthcare Information Network, Charlbury, UK

Acknowledgements We are grateful to the Healthcare Information For All (HIFA) network for their valuable advice, John Eyers for advising on and conducting the database searches, and to Tom Burnham, Sarah Cavanagh, Rita Reyburn and Lorenzo Moja for their comments on the protocol; Shilpa Maiya, Lisa Yamasaki, Yuka Arakura, Reina Takeda and HIFA volunteers for helping with screening. This review contributes to the broader effort of the HIFA working group on information for prescribers and users of medicines, which is striving for 'A world where every prescriber and user of medicines will have access to independent, reliable, understandable information on the full range of commonly prescribed medicinesand will know where to find it'.

Contributors CS and MHvV jointly wrote the review. MHvV and MB conducted the searches. All the authors screened the references. MHvV and NDT extracted the data and assessed the quality of included studies. CS and NPW provided extensive comments on the review. All authors reviewed the final draft of the review.

Funding This work was supported by the Japanese Society for the Promotion of Science (JSPS) and the Healthcare Information For All (HIFA) network.

Disclaimer JSPS had no role in this designing and undertaking this systematic review. HIFA helped with the collection, analysis, interpretation of data and in the writing of the report. HIFA had no influence on the study design or in the decision to submit the paper for publication. The corresponding author confirms that he had full access to all the data in the study and had final responsibility for the decision to submit for publication.

Competing interests None declared.

Patient and public involvement Patients and/or the public were involved in the design, or conduct, or reporting, or dissemination plans of this research. Refer to the Methods section for further details.

\section{Patient consent for publication Not required.}

Ethics approval No ethics approval was required. The review will inform efforts that aim to improve the prescribing practices of healthcare workers in low and middle-income countries. Findings were disseminated through the Healthcare Information For All network.

Provenance and peer review Not commissioned; externally peer reviewed.

Data availability statement Data sharing is not applicable as no data sets were generated and/or analysed for this study.

Open access This is an open access article distributed in accordance with the Creative Commons Attribution Non Commercial (CC BY-NC 4.0) license, which permits others to distribute, remix, adapt, build upon this work noncommercially, and license their derivative works on different terms, provided the original work is properly cited, appropriate credit is given, any changes made indicated, and the use is non-commercial. See: http://creativecommons.org/ licenses/by-nc/4.0/.

\section{ORCID iDs}

Chris Smith http://orcid.org/0000-0001-9238-3202

Nguyen Hai Nam http://orcid.org/0000-0001-5184-6936

\section{REFERENCES}

$1 \mathrm{WHO}$. The world medicines situation 2011 rational use of medicines. Available: http://apps.who.int/medicinedocs/documents/s18064en/ s18064en.pdf

2 Krause G, Borchert M, Benzler J, et al. Rationality of drug prescriptions in rural health centres in Burkina Faso. Health Policy Plan 1999;14:291-8

3 Antimicrobial resistance WHO. Available: http://www.who.int/ mediacentre/factsheets/fs194/en/

4 Pakenham-Walsh N, Bukachi F. Information needs of health care workers in developing countries: a literature review with a focus on Africa. Hum Resour Health 2009;7:30.

5 Salmasi S, Khan TM, Hong YH, et al. Medication errors in the Southeast Asian countries: a systematic review. PLoS One 2015;10:e0136545

6 Hussain A, Bukhari NI, Hussain K, et al. Preliminary assessment of the prescribing errors in outpatient department of public sector teaching hospitals in Lahore. Healthmed 2011;5:101-5.

7 Park E, Masupe T, Joseph J, et al. Information needs of Botswana health care workers and perceptions of wikipedia. Int J Med Inform 2016;95:8-16.

8 Pakenham-Walsh N, Priestly C, Smith R. Meeting the information needs of health workers in developing countries. BMJ 1997;314:90.

9 Jafar TH, Jessani S, Jafary FH, et al. General practitioners' approach to hypertension in urban Pakistan: disturbing trends in practice. Circulation 2005;111.

10 Vasan A, Mabey DC, Chaudhri S, et al. Support and performance improvement for primary health care workers in low- and middleincome countries: a scoping review of intervention design and methods. Health Policy Plan 2017;32:437-52.

11 Smith $\mathrm{C}$, van Velthoven $\mathrm{MH}$, Pakenham-Walsh N. How do primary healthcare workers in low-income and middle-income countries obtain information during consultations to aid safe prescribing? A systematic review protocol. BMJ Open 2019;9:e023015.

12 Higgins JPT GS. Cochrane Handbook for systematic reviews of interventions version 5.1.0, 2011. Available: www.cochranehandbook.org [Accessed Mar 2011].

13 CRD. Systematic reviews: centre for reviews and dissemination (CRD) guidance for undertaking systematic reviews in health care, 2010. Available: www.york.ac.uk/inst/crd/index_guidance.htm

14 Shamseer L, Moher D, Clarke M, et al. Preferred reporting items for systematic review and meta-analysis protocols (PRISMA-P) 2015: elaboration and explanation. BMJ 2015;349:g7647 http://www.bmj com/content/349/bmj.g7647.abstract

15 Declaration of Alma-Ata: International Conference on primary health care. Alma-Ata, USSR, 1978. Available: http://www.who.int/ publications/almaata_declaration_en.pdf

16 Sterne JA, Hernán MA, Reeves BC, et al. ROBINS-I: a tool for assessing risk of bias in non-randomised studies of interventions. BMJ 2016;355:i4919.

17 CASP. Critical appraisal skills programme. CASP checklist: 10 questions to help you make sense of a qualitative research, 2018. Available: https://casp-uk.net/wp-content/uploads/2018/01/CASP Qualitative-Checklist-2018.pdf

18 Downes MJ, Brennan ML, Williams HC, et al. Development of a critical appraisal tool to assess the quality of cross-sectional studies (axis). BMJ Open 2016;6:e011458.

19 Udezi WA, Oparah AC, Enyi KU. An investigation of drug information needs of Nigerian pharmacists. Drug Inf J 2007;41:471-9.

20 Wabe NT, Raju NJ, Angamo MT. Knowledge, attitude and practice of patient medication counseling among drug dispensers in North West Ethiopia. J Appl Pharm Sci 2011;1:85-90.

21 Asmelashe Gelayee D, Binega Mekonnen G, Birarra MK. The needs and resources of drug information at community pharmacies in Gondar town, Northwest Ethiopia. Biomed Res Int 2017;2017:1-6.

22 Kamuhabwa AAR, Kisoma S. Factors influencing prescribing practices of medical practitioners in public and private health facilities in Dar ES Salaam, Tanzania. Trop. J. Pharm Res 2015;14:2107-13.

23 Samiak L, Vince JD. The use of the paediatric standard treatment book by clinic and health centre staff. P N G Med J 2000;43:69-75.

24 Usanga L, Gavaza P, Matema S, et al. Information technology use in community pharmacies in Harare, Zimbabwe. East Cent African $J$ Pharm Sci 2007;10:45-9.

25 Agbo HA, Madaki AJK, Envuladu EA. Exploring the prevalence of malaria and prescribing pattern of antimalarial treatment at an urban primary health care centre. Jos J Med 2012;6:59-62.

26 Oduor E, Pang C, Wachira C, et al. Exploring rural community practices in HIV management for the design of technology for hypertensive patients living with HIV 2019. 
27 Reynolds J, Wood M, Mikhail A, et al. Malaria "diagnosis" and diagnostics in Afghanistan. Qual Health Res 2013;23:579-91.

28 Perwaiz lqbal S, Rahman M. Challenges faced by general practitioners in Pakistan in management of tuberculosis: a qualitative study. Rawal Med J 2013;38:249-52.

29 Graham K, Sinyangwe C, Nicholas S, et al. Rational use of antibiotics by community health workers and caregivers for children with suspected pneumonia in Zambia: a cross-sectional mixed methods study. BMC Public Health 2016;16:897.

30 Anasi SN, Lawal FO, Paul-Ozieh A. Use of Internet as health information resource by community pharmacists in Nigeria. Information and Learning Science 2018;119:545-54.

31 Rusatira JC, Tomaszewski B, Dusabejambo V, et al. Enabling access to medical and health education in Rwanda using mobile technology: needs assessment for the development of mobile medical educator Apps. JMIR Med Educ 2016;2:e7.

32 Rambaud-Althaus C, Shao A, Samaka J, et al. Performance of health workers using an electronic algorithm for the management of childhood illness in Tanzania: a pilot implementation study. Am J Trop Med Hyg 2017;96:249-57.

33 Shao AF, Rambaud-Althaus C, Swai N, et al. Can smartphones and tablets improve the management of childhood illness in Tanzania? A qualitative study from a primary health care worker's perspective. BMC Health Serv Res 2015;15:135.

34 Bessat C, Zonon NA, D'Acremont V. Large-Scale implementation of electronic integrated management of childhood illness (elMCl) at the primary care level in Burkina Faso: a qualitative study on health worker perception of its medical content, usability and impact on antibiotic prescription and resistance. BMC Public Health 2019;19:449.

35 Segal JB, Arevalo JB, Franke MF, et al. Reducing dosing errors and increasing clinical efficiency in Guatemala: first report of a novel $\mathrm{mHealth}$ medication dosing APP in a developing country. BMJ Innov 2015;1:111-6.

36 Catalani C, Green E, Owiti P, et al. A clinical decision support system for integrating tuberculosis and HIV care in Kenya: a humancentered design approach. PLoS One 2014;9:e103205.
37 Abouda M, Hamzaoui A, Drira E, et al. The effect of an integrated syndromic respiratory disease guideline in primary health care settings. J Eval Clin Pract 2015;21:976-81.

38 Adams JL, Almond MLG, Ringo EJ, et al. Feasibility of nurse-led antidepressant medication management of depression in an HIV clinic in Tanzania. Int J Psychiatry Med 2012;43:105-17.

39 Ngongoni RF, Gan G, Deng Y, et al. Prescribing and dispensing practices for medicines used to treat non-communicable diseases in Uganda: a cross-sectional study. The Lancet Global Health 2018;6:S23.

40 Forsberg BC, Montagu D, Sundewall J. Moving towards in-depth knowledge on the private health sector in low- and middle-income countries. Health Policy Plan 2011;26 Suppl 1:i1-3.

41 Mills A, Brugha R, Hanson K, et al. What can be done about the private health sector in low-income countries? Bull World Health Organ 2002;80:325-30.

42 Peabody JW, Taguiwalo MM, Robalino DA, et al. Improving the Quality of Care in Developing Countries. In: Jamison DT, Breman JG, Measham AR, et al, eds. Disease control priorities in developing countries. 2nd edn. Washington (DC: World Bank, 2006.

43 WHO. Who global patient safety challenge: medication without harm, 2017. Available: http://www.who.int/patientsafety/medicationsafety/medication-without-harm-brochure/en/

44 Teng T-HK, Tromp J, Tay WT, et al. Prescribing patterns of evidencebased heart failure pharmacotherapy and outcomes in the ASIANHF registry: a cohort study. Lancet Glob Health 2018;6:e1008-18.

45 WHO. Who model list of essential medicines, 21th list, 2019. Available: https://www.who.int/medicines/publications/ essentialmedicines/en/

46 Palazuelos D, Diallo AB, Palazuelos L, et al. User perceptions of an $\mathrm{mHealth}$ medicine dosing tool for community health workers. JMIR Mhealth Uhealth 2013;1:e2.

47 Praveen D, Patel A, Raghu A, et al. SMARTHealth India: development and field evaluation of a mobile clinical decision support system for cardiovascular diseases in rural India. JMIR Mhealth Uhealth 2014;2:e54. 